\title{
Fourier-domain lightfield microscopy: a new paradigm in 3D microscopy
}

Sánchez-Ortiga, Emilio, Scrofani, Gabriele, MartinezCorral, Manuel, Saavedra, Genaro

Emilio Sánchez-Ortiga, Gabriele Scrofani, Manuel Martinez-Corral, Genaro Saavedra, "Fourier-domain lightfield microscopy: a new paradigm in 3D microscopy," Proc. SPIE 11521, Biomedical Imaging and Sensing Conference 2020, 115210B (15 June 2020); doi: 10.1117/12.2573221

SPIE Event: SPIE Technologies and Applications of Structured Light, 2020, Yokohama, Japan 


\title{
Fourier-domain lightfield microscopy: a new paradigm in 3D microscopy
}

\author{
Emilio Sanchez-Ortiga, Gabriele Scrofani, Manuel Martinez-Corral, Genaro Saavedra* \\ 3D Imaging and Display Laboratory, Universitat de València, E46100 Burjassot, Spain
}

\begin{abstract}
Recently, integral (also known as lightfield or plenoptic) imaging concept has been applied successfully to microscopy. The main advantage of lightfield microscopy when compared with conventional 3D imaging techniques is that it offers the possibility of capturing the 3D information of the sample after a single shot. However, integral microscopy is now facing many challenges, like improving the resolution and depth of field of the reconstructed specimens or the development and optimization of specially-adapted reconstruction algorithms. This contribution is devoted to review a new paradigm in lightfield microscopy, namely, the Fourier-domain integral microscope (FiMic), that improves the capabilities of the technique, and to present recent advances and applications of this new architecture.
\end{abstract}

Keywords: Fourier lightfield microscopy, optical sectioning, real-time three-dimensional microscopy, phase imaging.

*genaro.saavedra@uv.es; phone: +34963544343

\section{Introduction}

Since Gabriel Lippmann, in its seminal work ${ }^{1}$, reported in 1908 a new technique, named as Integral Photography, to capture perspective information of $3 \mathrm{D}$ scenes in a single shot, the integral (also known as plenoptic or lightfield) concept has been evolving following the progress of available recording and display devices. In particular, integral imaging was applied to microscopy first by Jang and Javidi $^{2}$ aiming to display the microscopic images and by Levoy et al. ${ }^{3}$ adapting to microscopy the concept of plenoptic imaging. Currently, however, conventional lightfield microscopy faces many challenges, due especially to the inherent tradeoffs the technique suffers regarding resolution and depth of field of the reconstructed images. While some researchers are focusing their efforts in exploring new deconvolution or interpolation tools or even some potential improvement by time multiplexing, a fully optical solution has been proposed that simultaneously solves problems of vignetting and alleviates the above limitations in resolution and depth of field. The key point of the proposal is to place a microlens array (MLA) at the Fourier plane, namely, the aperture stop of a microscope objective, instead of the neighborhood of the image plane ${ }^{4}$. This Fourier-domain integral microscopy (FiMic) has proven to be a very efficient alternative to conventional lightfield microscopy, providing an extended depth of field and enhanced lateral resolution. ${ }^{5}$ In this contribution we review this new paradigm in integral microscopy, presenting the state-of-the-art of the technique and showing the future challenges that FiMic should address.

\section{Fourier-domain lightfield microscopy}

\section{FiMic arquitecture}

The optical layout of a typical FiMic setup is shown in Fig. 1. It is composed of five optical elements: the microscope objective (MO), the relay lenses RL1 and RL2 (in a telecentric configuration), the MLA and the field-stop (FS). The MLA should be placed at the aperture diaphragm (AD) of the MO. However, since this aperture stop is not typically accessible in conventional MOs and the MLAs available in the market are limited, the final design conjugates the AD and the MLA by means of the telecentric relay RL1+RL2. The FS is used to limit the field of view to avoid overlapping between the different elemental images (EIs) registered at the sensor plane, just behind each microlens of the MLA.

Following the integral imaging concept, the detector (CCD in Fig. 1) captures the spatio-angular information at the MLA plane, namely, the AD plane, also known as

Biomedical Imaging and Sensing Conference 2020, edited by T. Yatagai, Y. Aizu, O. Matoba, Y. Awatsuji, Y. Luo,

Proc. of SPIE Vol. 11521, 115210B · @ 2020 SPIE · CCC code: 0277-786X/20/\$21 · doi: 10.1117/12.2573221 
Fourier plane. This location is completely different from the placement of the MLA in conventional integral microscopy, providing now a different orthographic perspective of the sample on each EI. Resolution and depth of field are improved dramatically respect to those conventional lightfield microscopes. ${ }^{5}$ However, FiMic originally shared with them some drawbacks, such as the lacking of optical sectioning capability or the inability to image transparent objects. Some progresses have been reported recently for overcoming these limitations, which are presented in the next two sections.

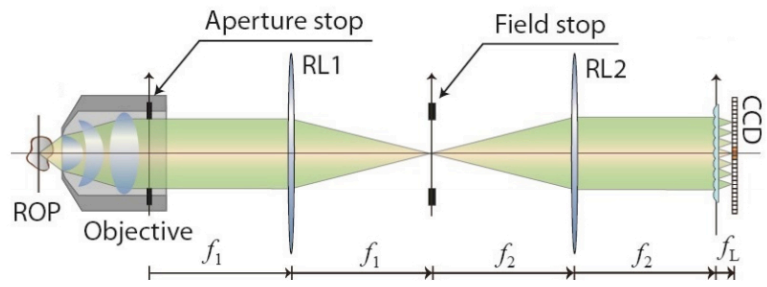

Figure 1. Optical layout of a typical FiMic system.

\section{Optical-sectioning imaging by FiMic}

The spatio-angular information captured in any lightfield imaging system can be exploited in several ways. Among them, computational refocusing of the scene to different depth planes can be carried out. Several computational algorithms for refocusing to different depth planes have been reported but most of them provides refocused depth images lack of optical sectioning when using conventional algorithms. This happens because any computed depth image integrates rays proceeding from the entire $3 \mathrm{D}$ scene. Then, out-offocus light is not rejected, and the refocused images have a misty appearance, with worsened contrast. This lack of optical sectioning is a crucial problem in 3D microscopy, hindering the proper reconstruction of 3D (thick) samples. A first attempt to overcome this limitation was based on the 3D deconvolution between the stack of refocused depth images and a synthetic 3D point spread function ${ }^{6}$. This algorithm, however, had the drawback of requiring long calculation time, and of providing important background noise typically inherent to deconvolution procedures. Recently, a new protocol for obtaining optically-sectioned 3D images in real time has been reported ${ }^{7}$. This procedure overcomes the drawbacks described above, and it is well suited for the case of fluorescent, sparse, 3D microscopic samples. Although the procedure is designed for its direct application to the orthographic views captured with a FiMic architecture, it could also be applied to the sub-images that are computed from the micro-images captured with a conventional lightfield microscope.

\section{Imaging phase objects by means of FiMic}

A very wide field of research in microscopy is for objects that have an extremely low contrast or that are completely transparent. Classical techniques as dark-field or phase contrast imaging are routinely used to increase the visibility of the images of these transparent (phase) samples. However, the extension those techniques to thick (3D) phase specimens has been a big issue for long time. Some advances in solving this limitation has been proposed recently by using the combination of FiMic and a proper illumination of the 3D sample. ${ }^{8,9}$ Thus, in order to obtain a $3 \mathrm{D}$ reconstruction of a transparent sample the use of a FiMic with a dark-field illumination can be utilized. In this way a $3 \mathrm{D}$ reconstruction of a phase object is achieved.

\section{Results and Discussion}

In Fig. 2 an example of the results that can be achieved with the application of the new optical-sectioning algorithm above mentioned ${ }^{7}$ is shown. Following the scheme shown in Fig. 1, the used FiMic consisted of an infinite-corrected MO $(20 \times, N A=0.5)$, an optical relay composed of two achromats with focal lengths of 200 $\mathrm{mm}$ and $100 \mathrm{~mm}$ (for R1 and R2, respectively), and a MLA with focal length of $6.5 \mathrm{~mm}$ and pitch $1 \mathrm{~mm}$ (hexagonal array). The camera employed had $2560 \times 1920$, $2.2 \mu \mathrm{m}$ square pixels. The integral image of a 3D sample consisting of a number of fluorescent beads of sizes ranging for 10 to $100 \mu \mathrm{m}$ in water was obtained. A set of 25 refocused depth planes was obtained after the application of the conventional refocusing (CR) algorithm $^{3}$, the deconvolution-based refocusing ${ }^{6}$ (DBR) scheme, and the optical-sectioning refocusing ${ }^{7}$ (OSR) technique. After the application of a maximum intensity projection algorithm, three $3 \mathrm{D}$ renders were obtained. Three orthographic perspectives of these 3D images are shown in Fig. 2 when using an integral image composed 
of 12 EIs captured by the sensor. Note that the opticalsectioning capability of the OSR algorithm is clear, since the images of the beads are almost free of out-of-focus light in this case.

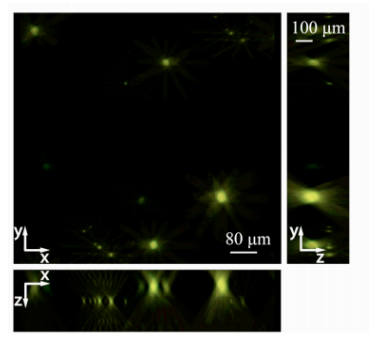

(a)

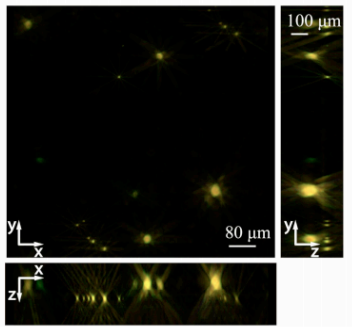

(b)

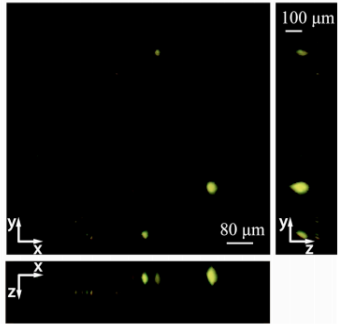

(c)
Figure 2. Views of the reconstructed $3 \mathrm{D}$ render from a FiMic single shot computed by using CR (a), DBR (b), and OSR (c) algorithms. The sample is a set of fluorescent beads as explained in main text.

By utilizing the above FiMic setup, but using a different $\mathrm{MO}(4 \times, N A=0.1)$, the results shown in Fig. 3 were obtained. In this case, the specimen was a Petri dish partially filled with a mixture of high-viscosity transparent liquid soap and water. The dish content was carefully stirred to generate a set of small air bubbles embedded in the liquid phase. A typical dark-field (annular) illumination was used adapted to the abovementioned MO. The same procedure as before was applied to obtain the reconstructed perspectives shown in Fig. 3. Only the results obtained with the OSR algorithm are presented there. It is interesting to note the good quality of the 3D reconstruction of the transparent bubbles, providing the optical sectioning and the spatial resolution achieved with the combination of the OSR technique and the dark-field FiMic setup.
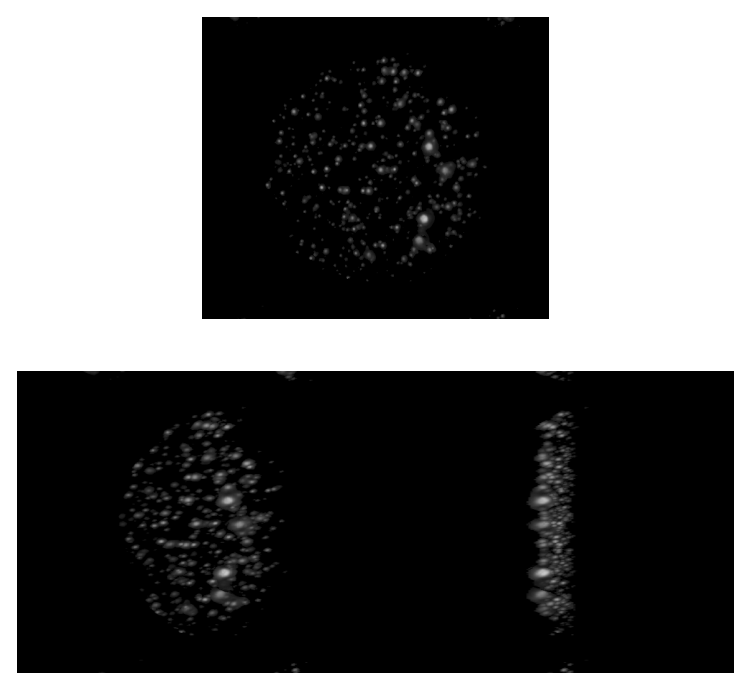

Figure 3. Views of the reconstructed 3D render from a dark-field FiMic single shot computed by using OSR algorithm. The sample is a set of transparent bubbles as explained in main text.

Acknowledgement: The authors acknowledge funding from Ministerio de Ciencia e Innovación, Spain (Grant RTI2018-099041-B-I00) and Generalitat Valenciana (Grant PROMETEO/2019/048).

\section{References}

[1] Lippmann, G., "Epreuves reversibles donnant la sensation du relief," J. Phys. Theor. Appl. 7(1), 821-825 (1908).

[2] Jang, J.-S. and Javidi, B., "Three-dimensional integral imaging of micro-objects," Opt. Lett. 29(11), 1230-1232 (2004).

[3] Levoy, M., Ng, R., Adams, A., Footer, M., and Horowitz, M., "Light field microscopy," ACM Trans. Graph. 25, 924-934 (2006).

[4] Llavador, A., Sola-Pikabea, J., Saavedra, G., Javidi, B., and Martinez-Corral, M., "Resolution improvements in integral microscopy with Fourier plane recording," Opt. Express 24(18), 20792-20798 (2016).

[5] Scrofani, G., Sola-Pikabea, J., Llavador, A., SanchezOrtiga, E., Barreiro, J.C., Saavedra, G., Garcia-Sucerquia, J., and Martinez-Corral, M., "FiMic: design for ultimate 3D-integral microscopy of in-vivo biological samples," Biomed. Opt. Express 9, 335-346 (2018). 
[6] Sánchez-Ortiga, E., Llavador, A., Saavedra, G., García-Sucerquia, J., and Martinez-Corral, M., "Optical sectioning with a Wiener-like filter in Fourier integral imaging microscopy,” Appl. Phys. Lett. 113(21), 214101 (2018).

[7] Sanchez-Ortiga, E., Scrofani, G., Saavedra, G., and Martinez-Corral, M., "Optical sectioning microscopy through single-shot lightfield protocol," IEEE Access (accepted, pending publication) (2020). doi: 10.1109/ACCESS.2020.2966323.

[8] Sola-Pikabea, J., Scrofani, G., Llavador, A., Barreiro, J.C., Martínez-Corral, M., and Saavedra, G., "Dark-field imaging with an integral microscope," in Proc. Focus on Microscopy 2017. Bordeaux, France, April 9-12, 2017. http://www.focusonmicroscopy.org/2017/PDF/1077_Sola -Pikabea.pdf.

[9] Scrofani, G., Sola-Pikabea, J., Sánchez-Ortiga, E., Martínez-Corral, M., and Saavedra, G., "Light-field Fourier Microscope (FiMic) for transparent objects," in Proc. Three-Dimensional Imaging, Visualization, and Display 2020, 11402-3. Anaheim, CA, USA, April 26-30, 2020. 